\title{
Visual cortex local field potential (LFP) in the mouse are linked to respiration and visual stimulation
}

\author{
Ran Zhai ${ }^{1, a}$,Jun Hui Cui ${ }^{1, b^{*}}$, Hong Wei Ding ${ }^{1, \mathrm{c}}$, Long Wang ${ }^{1, \mathrm{~d}}$ \\ ${ }^{1}$ Department of Electronic Engineering, Information School, Yunnan University,Kunming, China

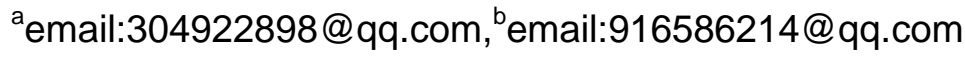

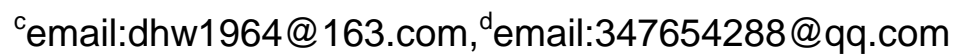

Keywords: Local field potential; Respiration; Visual stimulation; Multiple linear regression; Signal separation

\begin{abstract}
Current evidence suggests that local field potential activity in the whisker barrel cortex of awake mice is phase locked to respiration. In our study,we discuss the relevance among local field potential signals,respiration and visual stimulation.First,we obtain data from EEGs including two aspects:(1)the state of asleep;(2)the state of awake:(a)no visual stimulation;(b)a visual stimulus of Checkboard. Second,through some calculating models, such as:Double Exponential Smoothing Method, Multiple linear regression, Polynomial Nonlinear regression and so on,we can draw the conclusion that: visual cortex local field potential in the mice is related to respiration and visual stimulation, and the periodic changes of LFP have a significant impact on the periodic changes of respiration. Moreover,there is a positive correlation between the power spectrum of respiration and visual stimulation. In addition,basing on the Wavelet decomposition and reconstruction model, we separate the EEG components of the stimulus of Checkboard and respiration in awake state.
\end{abstract}

\section{Introduction}

Oscillatory neuronal activity is a common phenomenon in mammalian neocortex and can be readily observed in local field potential (LFP) and electroencephalogram signals. LFP signal is the comprehensive reflection of a large number dendritic potentials of local neurons in the brain . LFP associates with behavior controlled by brain,such as respiration and visual stimulation.

An electroencephalogram (EEG) is an amplification of electrical activity generated by neurons in the brain. EEG are recorded for two aspects and we can obtain three groups of data:(1)the state of asleep;(2)the state of awake:(a)no visual stimulation;(b)a visual stimulus of Checkboard. Additional,in order to study the effect of visual stimulation to LFP, we give a visual stimulus to mouse's eyes, when recording the LPF in the visual cortex area. The stimulation is a pair of Checkboards and the switching interval is random (the checkboard is placed in front of mouse, randomly opened or closed).

In our study,in order to discuss the relevance among local field potential signals, respiration and visual stimulation,we fit the obtained data through some fitting models.Moreover,we build a signal separation and reconstruction model, and separate the EEG signal relating to respiratory from asleep and awake LPF recordings .

\section{Material and Methods}

Animals. Experiments were performed on adult male C57BL/6J (B6) mice (48weeks old, 18-25 g body weight). Male mice were used to avoid variability in behavioural physiological measures related to oestrous cycle. All experiments were performed during the light cycle (between 1200 and 1700 hours). None of the mice had undergone any previous experimental procedure. All animal experimental procedures adhered to guidelines approved by the University of Tennessee Health Science Center Animal Care and Use Committee. Principles of laboratory animal care (NIH publication No. 86-23, rev. 1996) were followed. 
EEG recordings. For extracellular recordings, the guiding tubes of a computer-controlled microdrive (MiniMatrix, Thomas Recording, Germany) were lowered into the saline-filled recording chamber to a distance of less than $2 \mathrm{~mm}$ from the dural surface of the brain. The stainless steel guiding tubes also serve as reference electrodes and are eclectically connected to the brain tissue via the saline solution.Signals (LFP: local potential) are from visual cortex in the sleep or awake mouse. Then, 5 electrodes (glass insulated tungsten/platinum, impedance: 3.5-5.0MO)were slowly advanced through the intact dural into the visual cortex. The 5 electrode are recorded at the same time, each space of the electrode is $0.25 \mathrm{~mm}$. Electrode movements were controlled with micrometre resolution and digitally monitored. Local field potentials and spike signals were filtered (bandpass: 0.1 to $200 \mathrm{~Hz}$ ) using a hardware filter amplifier (FA32; Multi Channel Systems). Filtered and amplified voltage signals were digitized and stored on a computer hard disk (16 bit A/D converter;Sampling frequency: 1000 Hz;Data length: 30 seconds for LFP).

Data analyses and statistics. As previously described ,the original data of visual cortex local field potential (LFP) curve and Respiration curve in the mouse were examined by off-line analysis and spectral analysis was performed using MATLAB (Version R2013a).We use the existing models of Double Exponential Smoothing Method,Multiple linear regression,Polynomial Nonlinear regression, Wavelet decomposition and reconstruction to analyze and reconstruct the data respectively.

\section{Results}

According to the Secondary Exponential Smoothing model, we fit the obtained data of three groups, as shown in Fig. 1:

(a)

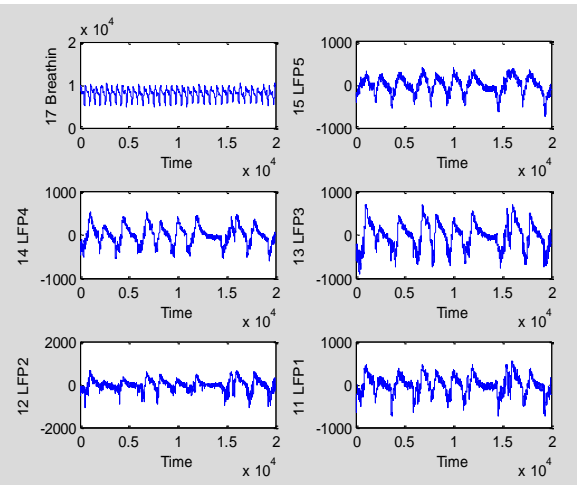

(c)

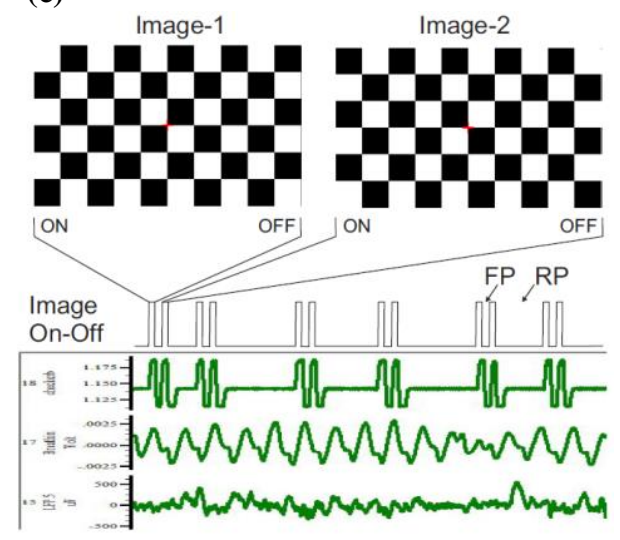

(b)

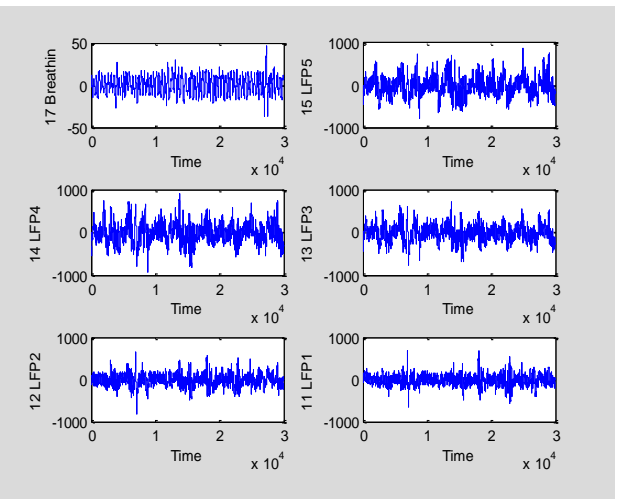

(d)

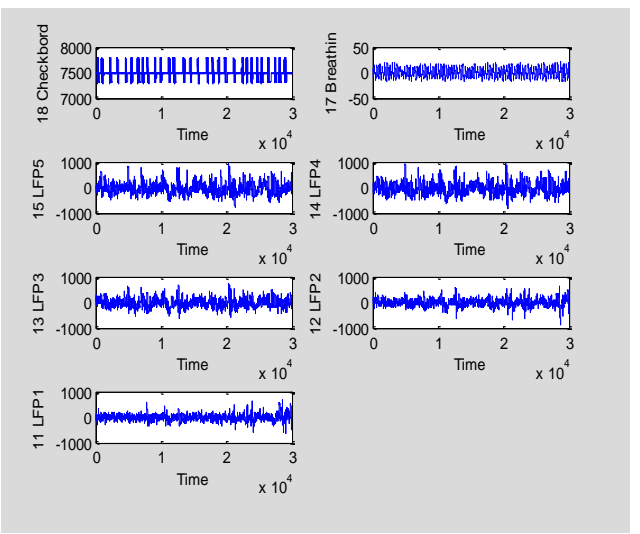

Fig. 1 Visual cortex local field potential (LFP) curve and Respiration curve in the mouse. Data from one mouse are shown, but results were reproduced in five out of five mice.Chanel 1-5(Ch11, Ch12, Ch13, Ch14, Ch15):Visual cortex local field potential (LFP) signals in the mouse;Chanel 6(Ch17):Respiration curve; Chanel 7(Ch18):two visual stimuli of CheckBoard.(a)the state of asleep;(b)the state of awake:no visual stimulation; (c)two visual stimuli of CheckBoard(FP: 
fixed-pause;RP: randomized-pause (0.2s - 2.0s).Image- 1 and Image-2 have the same size.Image does not move(Only "ON" and "OFF").The time of Image ON and the time of FP are not accurate;(d)the state of awake: a visual stimulus of Checkboard.

The correlation between Respiration and LFP during sleep. We establish a multiple linear regression model to analyze the correlation between LFP and respiration during sleep in mice.Set the dependent variable is $\mathrm{y}$, the independent variable is $\left(\mathrm{x}_{1}, \ldots, \mathrm{x}_{\mathrm{p}}\right)$, we obtain $\mathrm{n}$ groups of independent observation data $\left(\mathrm{y}_{\mathrm{i}} ; \mathrm{x}_{\mathrm{i} 1}, \ldots, \mathrm{x}_{\mathrm{ip}}\right), \mathrm{i}=1,2, \ldots, \mathrm{n}$. The multiple linear regression model is as follows:

$$
\left\{\begin{array}{l}
y_{i}=\beta_{0}+\beta_{1} x_{i 1}+\cdots+\beta_{p} x_{i p}+\varepsilon_{i}, i=1,2, \cdots n . \\
E\left(\varepsilon_{i}\right)=0, \\
D\left(\varepsilon_{i}\right)=0, \\
\varepsilon_{1}, \varepsilon_{2}, \cdots, \varepsilon_{i} \text { 相互独立. }
\end{array}\right.
$$

In many situations, we often assume that:

$$
\varepsilon_{i} \sim N\left(0, \sigma^{2}\right), i=1,2, \cdots, n \text {. }
$$

Through constant significant tests of regression equations and regression coefficients, we get the multiple linear regression equation reflecting the correlation between LFPs and respiration during sleep in mice:

$$
y_{A}=8018.3+0.3295 x_{3}-0.7034 x_{4}+0.2921 x_{5}
$$

The correlation between Respiration and LFP in the awake mouse. We establish a nonlinear polynomial regression model:

$$
y=\beta_{0}+\beta_{1} x+\cdots+\beta_{n} x^{n}
$$

First,take respiration(CH17) and $\mathrm{LFP}(\mathrm{Ch} 11 \sim \mathrm{Ch} 15)$ as $\mathrm{y}, \mathrm{x}$,respectively. We get the multiple linear regression equation reflecting the correlation between LFPs and respiration in the awake mouse:

$$
y=0.5620-0.0176 x_{1}+0.0202 x_{2}-0.0059 x_{3}+0.0066 x_{4}-0.0055 x_{5}
$$

\begin{tabular}{|c|c|c|c|c|c|}
\hline$F_{\alpha}(p, n-p-1) \quad \alpha$ & 0.1 & 0.05 & 0.025 & 0.01 & 0.005 \\
\hline$F_{\alpha}(5, \infty)$ & 1.85 & 2.21 & 2.57 & 3.02 & 3.35 \\
\hline$F_{\alpha}(1, \infty)$ & 2.71 & 3.84 & 5.02 & 6.64 & 7.88 \\
\hline
\end{tabular}

Second, use significant test of the value of $F$ and $F X$.From Table1, we know:F> $F_{\alpha}(5, \infty), F X>F_{\alpha}$ $(1, \infty)$ :The periodic changes of LFP( Ch11 Ch15) have a significant effect on the Respiration $(\mathrm{y})$.

Extraction and Separation of Effective EEG signal. We reconstruct a wavelet decomposition and reconstruction model to process and analyze EEG,and extract real EEG signals under the background of strong noise effectively.According to Multiple Linear Regression model,we get the corresponding multiple linear regression equations of EEG components relating to the stimulus of Checkboard and respiration respectively:

$$
\begin{aligned}
& y_{B}=7494.6-0.0617 x_{1}+0.0354 x_{2} \\
& y_{C}=0.6218-0.014 x_{1}+0.0072 x_{2}-0.0121 x_{3}+0.0082 x_{4}-0.0041 x_{5}
\end{aligned}
$$

By significance test of regression equation, we get that: $F>F_{\alpha}(\alpha$ is the given level of significance). 


\section{Discussion}

We establish a multiple linear regression model to analyze the correlation between LFP and respiration during sleep in mice.From Fig. 2 we can see:during sleep,the EEG relating to respiration have periodic rhythm, namely the periodic rhythm of EEG are related to respiration.

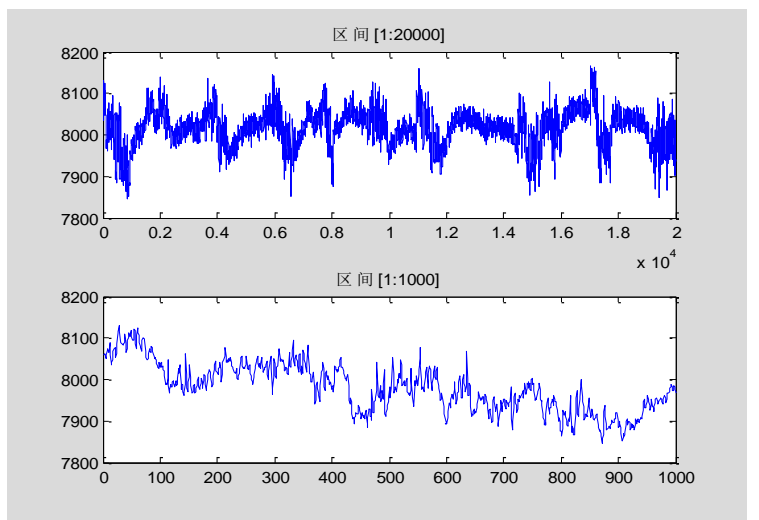

Fig. 2 Periodic rhythm of LFP related to respiration during sleep

Furthermore,we discussed the correlation between Respiration and LFP in the awake mouse.According to the two exponential smoothing model, we fit the curve of channel1 5 of LFP and curve of channel6 of respiration in the awake mouse to facilitate our observation, take the interval as[1:3000], as shown in Fig. 3(a),(b): Visual cortex local field potential and EEG corresponding with respiration all have periodic changes. In addition, we know: $\mathrm{F}>\mathrm{F} \alpha(5, \infty)$ and $\mathrm{FX}>\mathrm{F} \alpha(1, \infty)$, then we come to a conclusion :the periodic changes of LFP have a significant impact on the periodic changes of EEG corresponding with respiration,that is,respiration are multiple non-linear related to LFP in the awake mouse.

(a)

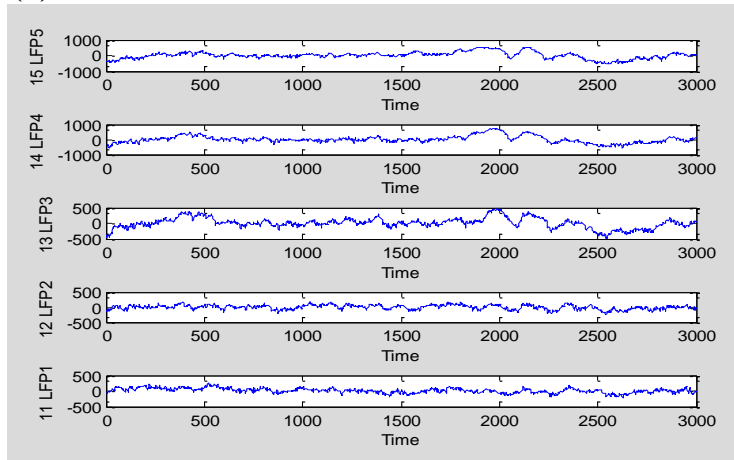

(b)

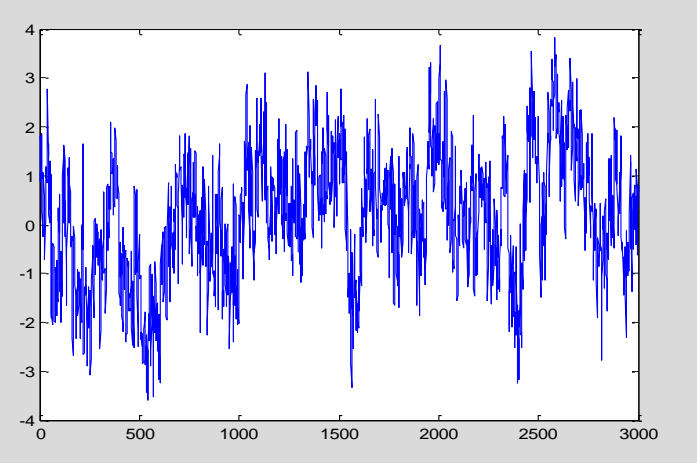

Fig. 3 Periodic changes of LFP and respiration in the awake mouse(a)LFP;(b)respiration.

In addition,we discussed the correlation between respiration and the visual stimulation. Then we can get the power spectrum curve of respiration and the visual stimulation.We can see:Power spectrum of respiratory curves has no significant correlation with Time curve of visual stimuli,while presents positive correlation with Power spectrum of visual stimuli .

On the basis of wavelet decomposition and reconstruction model,we separate the EEG components of the stimulus of Checkboard and respiration in awake state, as shown in Fig. 4.In order to analyze the relationship between the separated EEG components of the stimulus and the EEG of visual stimuli,we use the previous referred polynomial non-linear regression model.By significance test of regression equation, we get that: $\mathrm{F}>\mathrm{F} \alpha$ ( $\alpha$ is the given level of significance). So we can arrive at a conclusion: there is a polynomial nonlinear regression correlation between them. Beyond that,because the relationship is non-linear correlation between the two,we may infer that the components of EEG contain the factor of graphics. 
(a)

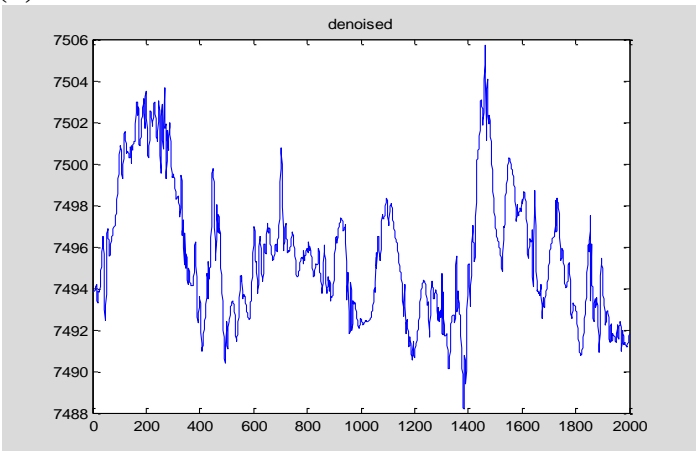

(b)

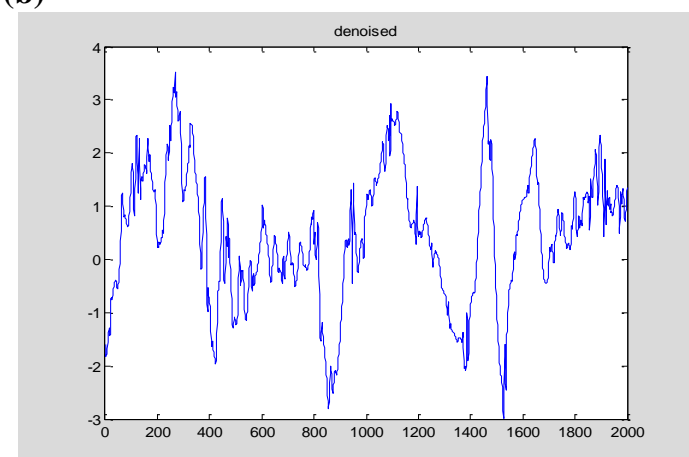

Fig. 4 The separated EEG components (a)the EEG components of the stimulus of Checkboard;(b)the EEG components of the stimulus of respiration.

In summary, by comparing the relevance among local field potential signals,respiration and visual stimulation on adult male mice, and using some calculating models, such as:Double Exponential Smoothing Method, Multiple linear regression and so on,we find clear indications: visual cortex local field potential in the mice is related to respiration and visual stimulation both in asleep and awake state.Furthermore,Visual cortex local field potential and EEG corresponding with respiration all have periodic changes, and the periodic changes of LFP have a significant impact on the periodic changes of respiration. Moreover,there is a positive correlation between the power spectrum of respiration and visual stimulation. In addition,we separate the EEG components of the stimulus of Checkboard and respiration in awake state,prove that there is a polynomial nonlinear regression correlation between them.Beyond that,because of the non-linear correlation between the two,we infer that the components of EEG contain the factor of graphics.It also confirms that: a combination of biological and mathematical may be form a good research.

\section{ACKNOWLEDGEMENT}

This work was supported by the National Natural Science Foundation of China 61461053.Natural Science Foundation of Yunnan Province (2010CD023); Graduate Scientific Research Fund of Yunnan University (ynuy201047) financial support of Yunnan University(No.XT412004).

\section{References}

[1] J. Ito,S. Roy,Y. Liu, M. Fletcher, L. Lu, J. D. Boughter,S. Grun, D. H. Heck, Whisker barrel cortex delta oscillations and gamma power in the awake mouse are linked to respiration,Nature Communication, 2014, April.

[2]Fu Y, Wang XS, Wang YC, Zhang J, Liang Z, Zhou YF, Ma YY. The effects of aging on the strength of surround suppression of receptive field of V1 cells in monkeys. Neuroscience doi: 10.1016/j.neuroscience.2010.05.015

[3]Peng Yuhua. Wavelet transform and engineering application [M]. Beijing: Science Press, 1999:13-51

[4]Si Shoukui et al, mathematical modeling algorithm and application of problem solving,Beijing: National Defence Industry Press, 2013.1:88

[5]Wu mengda et al, mathematical modeling tutorial, Beijing: Higher Education Press, 2011.8:136-139.

[6]Gong chun,MATLAB common algorithms collection,Beijing:Publishing House of electronics industry, 2008.6:522-523.

[7]Zheng Junli, Ying Qihang, Yang Weili. Signals and systems (Second Edition),Beijing: Higher Education Press, 2000:349-353. 